\title{
General Purpose Simulation System based on Excel Language
}

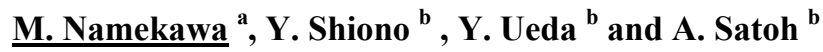 \\ ${ }^{a}$ College of Management and Economics, Kaetsu University, Kodaira Tokyo JAPAN \\ ${ }^{b}$ Faculty of Information Sciences and Arts, Toyo University, Kawagoe Saitama JAPAN
}

Email: mitsuhiro@namekawa.com

\begin{abstract}
:
Many kinds of simulation systems have been developed. All of them are general or special purpose simulation systems, and the latter are used especially in their specific fields. To carry out simulations using them, however, requires one to learn each method's original methods or conceptualizations. Naturally, there are simulations using general purpose language, such well, but it is difficult to build the computer model and also make its program. Moreover, professional knowledge concerning computer simulation is required to use them.

On the other hand, simulation techniques that enable one to perform a simulation by using general purpose languages, such as Excel and Octave, have attracted attention of researchers in many kinds of fields recently. The Excel language is a popular one for computer users and has the ability to process tables. The method is used to carry out simulation for simple systems with some queuing sub-systems connected in series, but not to do simulation for more complex systems such as network with queuing sub-systems connected in parallel. Therefore the advent of a method that is able to carry out easily simulation for more complex system has been desired.

Considering the above trend and background, we propose a new simulation system using Excel as a host language. This system, which we have been developing, makes it possible easily and simply to carry out simulations for network flow-type systems such as manufacturing systems, in which a certain kind of transaction moves in the network. Our system enables users to build the model of a network system visually, with queue sub-systems connected in series and/or parallel on the computer display through human interface. Therefore it is possible to use our simulation system not only for experts, but also for beginners in the field of computer simulation.
\end{abstract}

Our simulation system has the following characteristics:

1) Professional knowledge concerning simulation is not required for using it.

2) Knowledge of EXCEL language is not required for using it to perform fundamental simulations.

3) It is possible to carry out a simulation easily by only learning its method briefly.

4) It is possible for people, especially students with knowledge of the EXCEL language, to make complex simulations more simply and easily.

5) It is possible to represent and edit the simulation results freely using the functions of the Excel language

In this paper, we describe the approach of our simulation system, and the characteristics of management systems such as manufacturing processes. We also describe the functions and characteristics of our system. Finally, we verify the fundamental functions and performance of our system by carrying out a simulation using with our system

Keywords: simulation language, transaction flow-type system, manufacturing system, excel language 


\section{INTRODUCTION}

Many kinds of simulations have been carried out using various kinds of methods such as modeling and programming. They are roughly divided into two types of simulations. One is a general purpose language like C, two is a simulation system (Alan and Pritsker, 1995)(Chikada et al., 2010)( Geoffrey, 1978) or simulation language. All simulation systems require users to learn their own original world-views and grammars for carrying out a simulation using them.

On the other hand, simulation techniques that enable one to perform simulations using general purpose languages such as Excel have attracted the attention of researchers in many kinds of fields recently. The Excel language is a popular one for computer users, and has the characteristics to process tables. Excel is usually available to carrying out a queue system, solving to differential / difference equations. However, its method (Grenander, 1982)( Namekawa et al., 2005) is used to carry out simulations for simple systems with series queuing sub-systems, rather than simulations for more complex systems, such as a network with queuing sub-systems in parallel. Therefore, the advent of a method able to carry out simulations easily for more simplex systems using general purpose languages has been desired.

Considering the above trend and background, we propose a new simulation system using Excel as a host language. Simulation models are dived roughly into two models: the continuous model and discrete-event model. Our system is able to build both of these, but programming for the former is not simple. We call it SIMEX (SIMulation system by Excel language). This system is capable of carrying out simulations simply and easily for a network system with queue sub-systems connected in series and/or parallel in which a certain kind of transaction moves in a network, such as the production line of a manufacturing system. Our goal is that our system will enable users to build visually the model of a network system with queue sub-sytems connected in series and/or parallel on the computer display through human interface. Therefore, our simulation system will be accessible not only to experts, but also to beginners in the field of computer simulation

SIMEX has the following characteristics:

(1) Professional expertise in simulation is not required for using it. It will be preferable, however, for users to have expertise in analyzing queuing systems and solving differential / difference equations.

(2) Knowledge of the EXCEL language is not required for using it in case of fundamental simulations.

(3) It is possible to understand our system's approach quickly, and thereby to carry out simulations easily. It Users are required to analyze reasonably the system upon which they are going to carry out simulations.

(4) Our system is accessible for almost anyone, especially students with knowledge of the EXCEL language.

(5) It is possible to represent the simulation results freely by using the functions of the Excel language. Excel users are able to analyze the results of simulation satisfactorily through Excel's functions.

In this paper, we describe the characteristics of a management system such as the manufacturing process and economic system, in section 2; the approach of our simulation system in section 3; the functions and the characteristics of our system in section 4; and verification and validation by carrying out a simulation using our system in section 5 . Section 6 concludes the paper.

\section{CHARACTERISTICS OF MANUFACTURING SYSTEM}

Characteristics of the factory system include that it is easier to predict the outbreak interval between the product materials than with a general queue system. Moreover, it is enumerated that the flow of the element frequently repeats the divergence and the confluence there. This is to join it after junction in the process of manufacture and divergence by the identification of quality products, defective articles via product examination, and examination of adjusted products.

There is lost time that occurs as a result of the breakdown of the producing machine. It is understood that no device in a factory will always run normally; time passes, machine maintenance time is needed due to mechanical failure or worn-out parts.

Moreover, the problem of the stock control besides the queue at each stage of work is enumerated. Recently, Toyota's Kanban has widely utilized the so-called "just-in-time" system of production. Still, no stock was lost, and only the amount of Kanban is stocked. In addition, the stock known as "safety stock" used in case of 
breakdown or accident, is needed in the production. Furthemore, the "just-in-time" system of production of the cannot be arranged on all the production sites.

\section{WORLD-VIEW AND MODEL BUILDING OF OUR SIMULATION}

Our goal is that our simulation system enables user to build a model of a network system with some operation sub-systems connected in series and/or parallel in which a certain kind of transaction moves. We can build a model these operations process as a network to analyze some transactions (entities) flow. A manufacturing system is represented as a network flow-type system in which a certain kind of entities moves in the network as shown as Fig.1. The flow of these serial operations is built as a following network model. An operation generates a queue corresponding to the capacity of operation's ability. We will build this phenomenon as a queue sub-system in our network model. Arrival of units is built as a create model. Phenomenon that units go out of the manufacture is built as a terminate model. And the results of the simulation is specified in our network model as a statistics mode

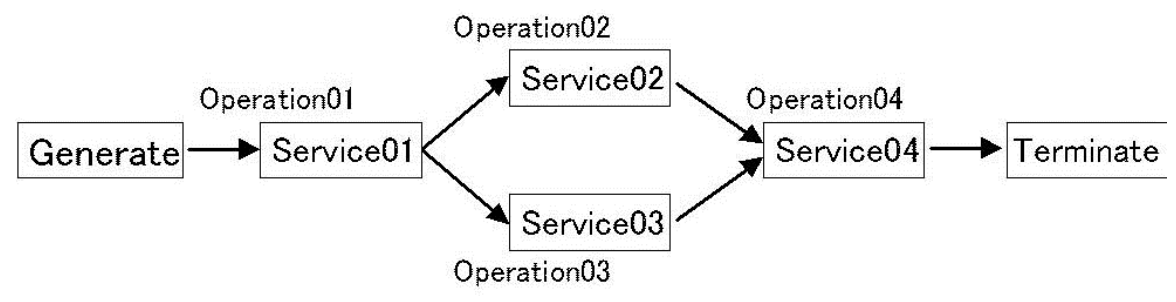

Figure 1. Schematic diagram of work stations in series.

\subsection{Network Representation Model}

We will construct a model of serial operations process in the manufacturing system of some products as a network model of our system. The facility of a manufacturer performs four operations; from service 01 to service02 as shown as Fig.1. These operations must be performed in series; for example, operation02 always follows operation01. And if in-house operations cannot gain access some of products, the operation of them is subcontracted as a company policy.

This example will illustrate the network modeling procedure which consists of identifying the entities to be modeled, constructing a graphical model of the entity flow process through system, and transcribing the graphical model into the SIMEX statement representation of the system. Specific network modeling concepts illustrated by this example include the creation of entities by a create node, the modeling of a service system with a queue node, and statistical collection at a statistics node.

\subsection{Queue Sub-system model}

To illustrate the basic concept of our system, we will construct a model of an inspection (operation) process in the manufacturing of some products. This process is a single resource queueing system. We use it as a node in a network model.

Queue system of an inspection process in the manufacturing is constructed by the followings:

(1) The arrival of product to the operation area.

(2) The buildup of products awaiting operation.

(3) The activity of operating product by a single operator.

The products are the system's entities. The operator (operation) is the resource and be modeled as a server. The server activity is the actual operaion, the buildup of the products awaiting service is the queue. These events are included in queue system of SIMEX as the queue node.

\subsection{Other Model}

(1) Create model for transactions(entities)

To generate the entity to the SIMEX, we must model the arrival of products to the system. Entities are inserted into a network by create model. It is required to specify the following characteristics in the create model. 
(a) The time at which the first entity is to be generated and sent into the network

(b) The time between generations of entities

(c) The maximum number of entities that can be generated at this model

(d) The maximum number of branches along which a created entity can be routed from this model

(2) Statistics model

Statistics can be collected on five types of variables at this model. Four of the variables refer to the time or times at which an entity arrives at statistics model (node). These variables are:
(a) Time-of-first arrival
(b) Time of all- arrivals
(c) Time between arrivals
(d) Interval statistics

(3) Terminate model for transaction(entity)

The terminate model is used to destroy or delete entities from the network. It can be used to specify the number of entities to be processed on a simulation run.

\section{FUNCTIONS AND THE CHARACTERISTICS OF OUR SYSTEM}

In the SIMEX, simulation is process with Excel function. Simulation modeling is to use the Excel function and VBA (Visual Basic for Application). Thus, it is enable the input such as detailed parameters. We construct the SIMEX to simple. So, It is clear, and it becomes easy to use.

About the modeling of the constitute simulation, we designed simply. So, if the user who is not used simulation, user operate the system easily.

User can input the SIMEX model on the display. Firstly, user forms a network model selecting and specifying parameters from such charactor as GENERATE, SERVER, STATISTIC and TERMINATE.

\subsection{Input the Simulation Model}

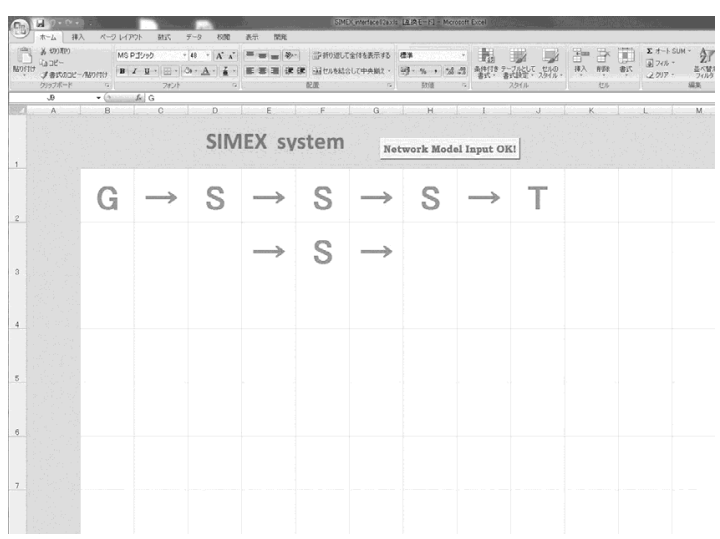

Fig. 2 shows the input display of the SIMEX. User input the SIMEX system in the middle of the display. If user will make the simulation model, user click the cell, and to choose the object. Fig. 3 show the user modeling. Then the object name of the simulation model is displayed on the window.

So, user can freely make the simulation model that thinks independently on the matrix.

Figure 2. Modelling Display of SIMEX system

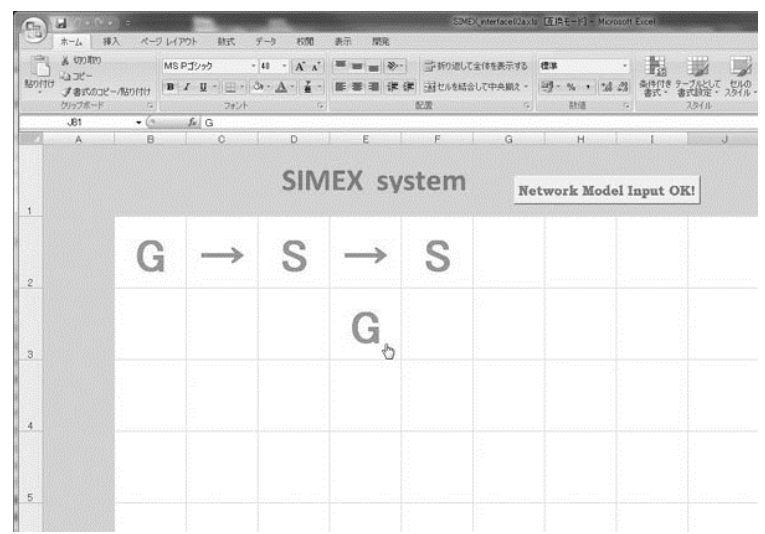

Figure 3. Select the Item for click of the cell. 


\subsection{Execution the simulation}

After the model is constructed as explained in the previous chapter, the simulation is executed. The macro of Excel is used for the execution of the simulation. After the model is constructed by the method where it explains by the previous paragraph, the simulation is executed. It must be use the macro (Visual Basic) which we execute the simulation of the Excel (Fig.4). The macro improves the high flexibility of the simulation.

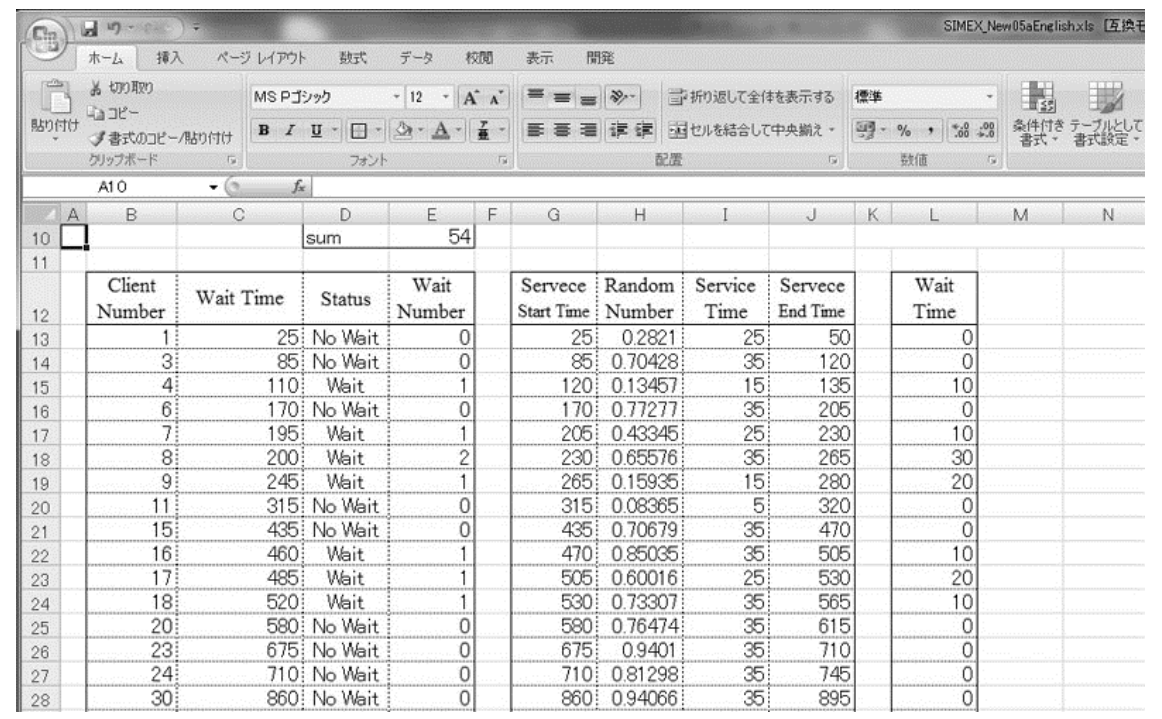

Figure 4. Execution of simulation.

\subsection{Output of the results}

The results of the simulation are divided into two : one is by Excel language, then two by the SIMEX output. The former is result of simulation through Excel language, that is original result in Excel table. User can analyze easily and simply simulation results such frequency tables and figures by processing the original excel table. Then the latter is shown as the followings:

(a) Statistics for variables based upon observation : Time in system, time in queue

(b) Queue statistics : queue number, average length, standard deviation, maximum length, current length, average waiting time

(c)Service activity statistics : average utilization, current utilization.

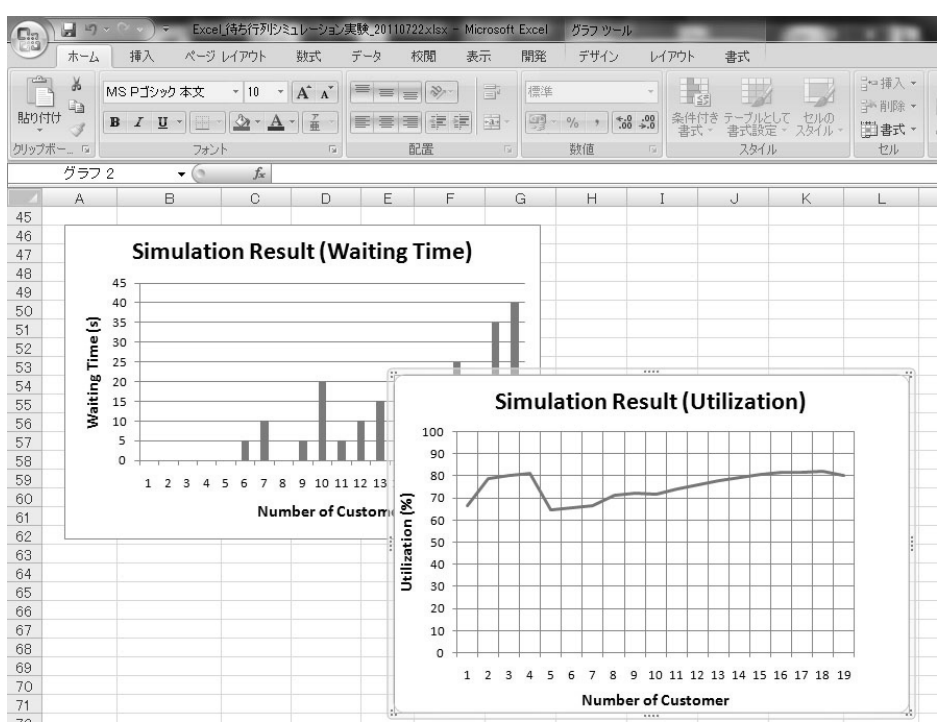

Figure 5. Display the simulation output(Excel). 


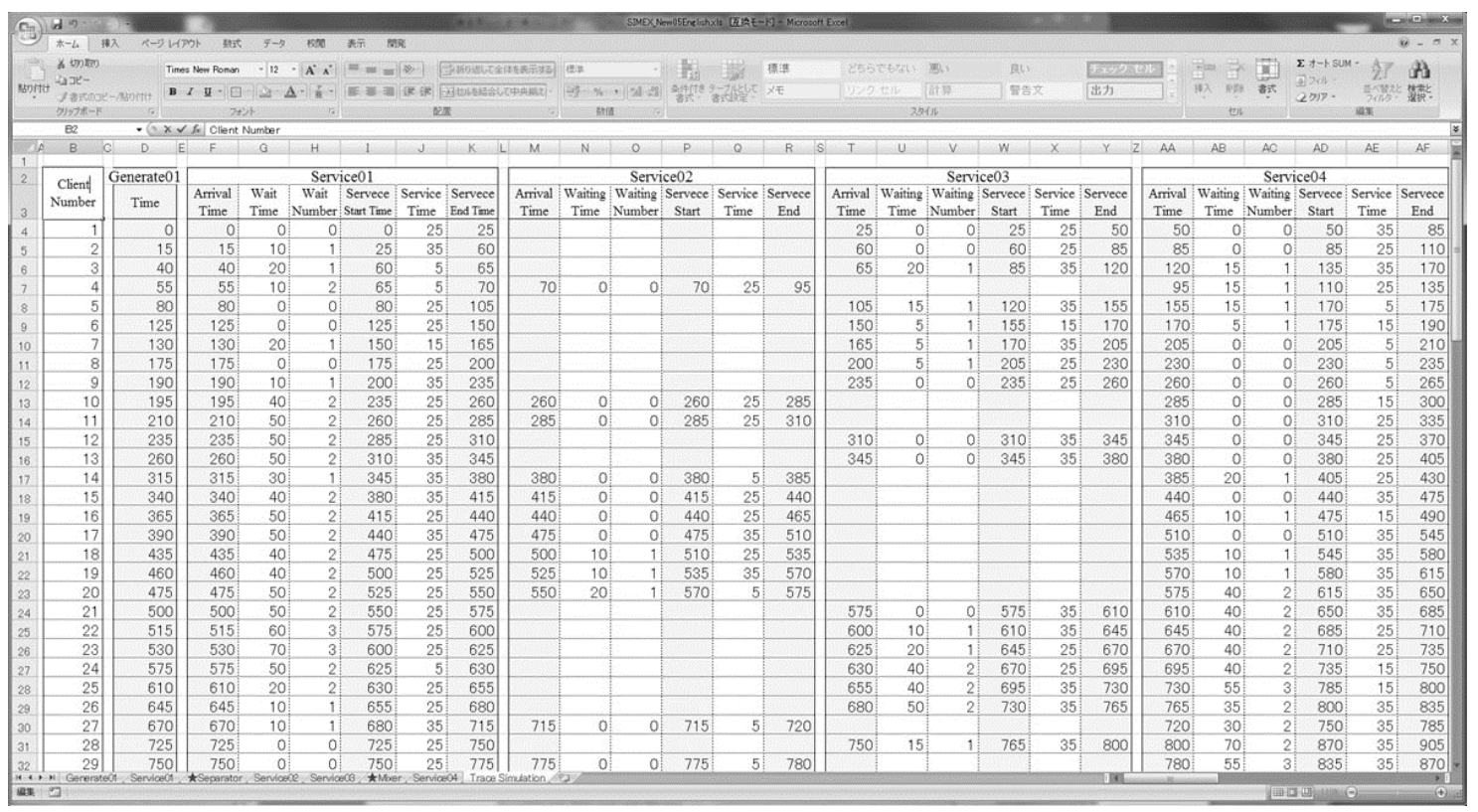

Figure 6. "Trace Simulation worksheet” of SIMEX.

This worksheet is intended to show simulation results in a list were made on each object., We know how the each element processed on the simulation. And it also to help the validate of simulation.

\section{VERIFICATION AND VALIDITY OF OUR SYSTEM}

We execute the simulation and verification of the simulation. We execute a simple queue model on the simulation system that we had constructed. As a result, it was able to be confirmed to operate firmly with the engine of excel. Figure 7 shows an execution result of the simulation. In this simulation, the situation in which 100 products flowed in the manufacturing process was modeled. And, it is the one that the lead time was measured. Because working hours is different because several kinds of products flow, we understand waiting time varies in one process. In this simulation result, it is understood that the waiting time of 50 seconds or less has gone out. Whether it is a value where this can be allowed as a production site can be examined. Thus, it is understood that our simulation system is very effective on the manufacturing system.

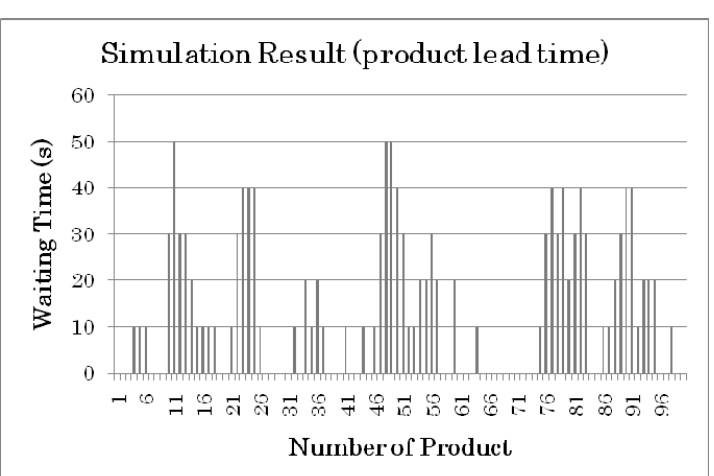

Figure 7. Output of the simulation (Excel).

\section{CONCLUDING REMARKS}

We have proposed a new simulation system (SIMEX) using Excel language as a host one. This is possible to carry out easily and simply simulations for a network flow-type system in which a certain kind of transaction moves in network such as a manufacturing system.

SIMEX enables user to build visually the model of a network system with queue sub-systems connected in series and/or parallel on the computer display through human interface. Moreover, it is possible to use our simulation system for not only expert, but also beginner of computer simulation.

We have been developing and verified the functions and performances of fundamental parts of our system now. Therefore we have not verified every functions and performances of a total system. We would like to establish to it at a near future. 


\section{REFERENCES}

Alan, A., Pritsker, B. (1995). Introduction to Simulation and SLAM II Fourth Edition. John Wiley \& Sons.

Chikada, N. , et.al. (2010). How to make the Action Game with Excel VBA, Impress Japan.

Geoffrey, G. (1978). SYSTEM SIMULATION second edition, Prentice Hall.

Grenander, U. (1982). Mathematical Experiments on the Computer. Academic Press.

Namekawa, M., F. Ueda, Y. Hioki, Y. Ueda and A. Satoh (2005), General Purpose Road Traffic Simulation System with a Cell Automaton Model, Proceedings of International Congress on Modelling and Simulation, 3002-3008.

Namekawa, M., F. Ueda, Y. Hioki, Y. Ueda and A. Satoh (2007), The Vehicle Junction Model and its Verification in Traffic Simulation, Proceedings of 2nd International Conference on Asian Simulation and Modeling 2007.

Neelamkkavil, F. (1987). Computer Simulation and Modelling. John Wiley \& Sons.

Satoh, J., M. Takahashi, M. Namekawa and A. Satoh (1992), Man-machine Interface of MITRAM, Proceedings of 12th Simulation Technology Conference, 149-152.

Shichijo,T., et.al. (2010). Excel VBA Programming, Softbank Creative. 